\title{
FATORES ASSOCIADOS A INDICADORES DE TRANSTORNOS MENTAIS EM CRIANÇAS E ADOLESCENTES: UMA REVISÃO INTEGRATIVA
}

\section{ASSOCIATED FACTORS WITH INDICATORS OF MENTAL DISORDERS IN CHILDREN AND ADOLESCENTS: AN INTEGRATIVE REVIEW}

\author{
João Mário Cubas ${ }^{1}$, Sofia Weidle Scatolin ${ }^{1}$, Deborah Ribeiro Carvalho ${ }^{1 *}$ \\ ${ }^{1}$ Pontifícia Universidade Católica do Paraná, Curitiba, Paraná Brasil \\ *Autor para contato: Programa de Pós-Graduação em Tecnologia em Saúde, Pontifícia \\ Universidade Católica do Paraná - Rua Imaculada Conceição, 1155. Bairro Prado Velho. \\ 80215-901. Curitiba. Paraná. E-mail: ribeiro.carvalho@pucpr.br
}

\section{RESUMO}

Crianças e adolescentes compõem um grupo vulnerável em relação a essa fase do desenvolvimento que merece atenção diferenciada. Atrelado a isso, existem fatores que podem contribuir diretamente no aparecimento de algum quadro de transtorno mental. Este artigo objetivou identificar fatores que estariam associados a indicadores de transtornos mentais em crianças e adolescentes. Foi realizada uma revisão integrativa da literatura científica de documentos publicados entre 2010 e 2015 nas bases de dados PsycINFO e LILACS e na biblioteca digital do Portal de Periódicos da Capes, com os descritores: "indicadores E crianças com transtorno mental", "indicadores E adolescentes com transtorno mental", "indicators AND children with mental disorder" $\mathrm{e}$ "indicators AND adolescents with mental disorder". Como resultado, foram apurados 20 indicadores relacionados a quadros de transtornos mentais na infância e na adolescência e 17 fatores a eles associados. Entre os principais indicadores identificados, o nível socioeconômico da família, associado à baixa renda, e a escolaridade parental, associada à baixa escolaridade dos pais, evidenciaram ser fatores de risco para quadros de transtornos mentais em crianças e adolescentes. Os resultados corroboraram os achados nos estudos que compuseram o corpus desta pesquisa, evidenciando a necessidade de implementação de políticas públicas destinadas à garantia de direitos de crianças e adolescentes.

Palavras-chave: indicadores, saúde mental, fatores de risco, criança, adolescente.

\begin{abstract}
Children and adolescents make up a vulnerable group in relation to this stage of development that deserves special attention. Attached to this, there are factors that can contribute directly to the onset of a mental disorder framework. This article aimed to identify factors associated with indicators of mental disorders in children and adolescents. An integrative review of scientific literature was held of documents published between 2010-2015, in PsycINFO and LILACS databases and Capes digital library with the descriptors: "indicadores $\mathrm{E}$ crianças com transtorno mental", "indicadores $\mathrm{E}$ adolescentes com transtorno mental", "indicators AND children with mental disorder" and "indicators AND adolescents with mental disorder". As a result it was identified 20 indicators related to mental disorders frameworks in childhood and adolescence and 17 factors associated with them. Among the main indicators identified, the socioeconomic status of the family associated with low income and parental education associated with low parental education have shown to be risk factors for mental disorders frameworks in children and adolescents. The results corroborate the findings in the studies that formed the corpus of this research, highlighting the need for public policies to guarantee the rights of children and adolescents.
\end{abstract}

Keywords: indicators, mental health, risk factors, children, adolescents. 


\section{INTRODUÇÃO}

Segundo dados da Organização Mundial da Saúde (OMS) (WHO, 2002), estima-se que 10\% a 20\% da população de crianças e adolescentes no mundo sofre com algum tipo de transtorno mental, sendo que metade das doenças mentais tem seu início até 14 anos de idade (WHO, 2004). Já no Brasil, a estimativa é de que cerca de $10 \%$ a $20 \%$ de crianças e adolescentes têm algum transtorno mental, dos quais aproximadamente $3 \%$ a $4 \%$ necessitam de tratamento intensivo com equipamentos de saúde mental (COUTO; DUARTE; DELGADO, 2008).

O surgimento de problemas relacionados à saúde mental ao longo do desenvolvimento do ser humano é influenciado por diversos precursores, que compreendem aspectos biológicos, sociais e psicológicos (FATORI DE SÁ; BORDIN; MARTIN, 2010). Na criança, as características pessoais, físicas e mentais, bem como o ambiente social em que ela vive, podem propiciar ameaças diretas ao seu desenvolvimento, podendo os transtornos mentais ocorrer também como consequência de problemas genéticos e biológicos adquiridos, como na presença de adversidades ambientais (MATSUKURA; FERNANDES, 2014). Nesse sentido, Fatori de Sá, Bordin e Martin (2010) relatam que os fatores de risco para o surgimento de um transtorno mental podem ser definidos como a presença de uma característica, experiência ou evento que venha a aumentar a probabilidade da ocorrência de determinado desfecho, quando comparado a uma população não exposta a esse fator.

São vários os fatores que tornam a criança e o adolescente mais suscetíveis ao desenvolvimento de algum problema relacionado à saúde mental. Avanci et al. (2007), em seu estudo, elencam: os individuais (sexo, idade e características psicológicas, como autoestima, autoconfiança e determinação); os familiares (histórico de transtornos mentais especialmente na mãe, problemas com álcool/drogas, casos de violência física, psicológica e sexual, violência entre os próprios genitores, perdas/luto e separação dos pais); os socioculturais (pobreza, violência no contexto social e falta de apoio/suporte social); e os biológicos. Um dos grandes desafios é entender e estudar a articulação desses fatores entre si e a forma como se relacionam com os comportamentos e a saúde (AVANCI et al., 2007).
Carvalho et al. (2011) indicam investigações para a análise de fatores pessoais e comportamentais associados aos indicadores de saúde mental. Nesse sentido, acrescentam que essas investigações são realizadas mediante o monitoramento de variáveis como o sentimento de tristeza, a dificuldade de dormir em virtude de preocupações, sentimentos de solidão, a ideação suicida e a relação destes com demais fatores relacionados à saúde. Afirmam, também, que adolescentes do sexo feminino que estudavam em período noturno eram mais vulneráveis, devendo ser priorizados nas intervenções (CARVALHO et al., 2011).

No entanto, pesquisas sobre a prevalência de indicadores de saúde mental em crianças e adolescentes são incipientes (CARVALHO et al., 2011), assim como pesquisas acerca de fatores de risco para o surgimento de transtornos mentais nesse público, sendo estas essenciais para o campo da saúde pública, pois o conhecimento desses fatores contribui no desenvolvimento de estratégias de intervenção em determinado público vulnerável (FATORI DE SÁ; BORDIN; MARTIN, 2010; AVANCI et al., 2007). No Brasil especificamente, verificam-se poucos estudos atinentes à temática, não somente acerca dos fatores relacionados com a saúde mental, como também da distribuição das psicopatologias e suas etiologias (AVANCI et al., 2007).

Frente à problemática apontada e à respectiva escassez de estudos, o objetivo deste artigo foi identificar fatores associados a indicadores de transtornos mentais em crianças e adolescentes, com vistas a disponibilizar novos elementos que levem à discussão para a implementação de políticas públicas destinadas à garantia de direitos desse público.

\section{METODOLOGIA}

Trata-se de uma revisão integrativa da literatura, a qual, segundo Mendes, Silveira e Galvão (2008), consiste na construção de uma análise mais ampla da literatura, contribuindo para discussões sobre métodos e resultados de pesquisas.

Para selecionar os estudos da revisão, foi utilizado o diagrama da metodologia Preferred Reporting Items for Systematic Reviews and Meta-Analyses (PRISMA) (LIBERATI et al., 2009), visando a identificar ao final do processo os documentos aptos para a discussão da temática (Figura 1). Foi realizada a 
seleção dos estudos primários, de acordo com o objetivo da pesquisa, de forma que atendessem aos critérios de inclusão previamente definidos (estudos publicados entre os anos de 2010 e 2015). Todos os documentos identificados por meio da estratégia de busca foram inicialmente avaliados mediante análise dos títulos e resumos. Optou-se pela busca nas seguintes bases de dados: PsycINFO, da American Psychological Association, e Literatura Latino-Americana e do Caribe em Ciências da Saúde (LILACS), como também na biblioteca digital do Portal de Periódicos da Coordenação de Aperfeiçoamento de Pessoal de Nível Superior (Capes). Como descritores, foram utilizados os seguintes termos: "indicadores E crianças com transtorno mental", "indicadores E adolescentes com transtorno mental", "indicators AND children with mental disorder" e "indicators AND adolescents with mental disorder". Não houve seleção quanto ao idioma e tipo de documento (artigo, tese, dissertação etc.), tampouco ao tipo de estudo (revisão sistemática, estudo longitudinal etc.).

A partir dos documentos selecionados, foram extraídas informações, sistematizadas em dois quadros, contemplando: a) autor/ano de publicação, país, descritores, tipo de estudo e resultados; b) indicadores relacionados e fatores a eles associados. O Quadro 2 foi construído com o intuito de apontar os indicadores e os fatores relacionados, fornecendo sua quantificação para a apresentação dos resultados e discussão.

\section{RESULTADOS}

A metodologia utilizada para seleção dos artigos pode ser verificada na Figura 1. No primeiro processo (identificação dos estudos), foram encontrados 571 artigos (304 no Portal de Periódicos da Capes, 14 no PsycINFO e 253 no LILACS); uma vez que houve repetição de muitos estudos, após a eliminação dos duplicados, restaram 331 estudos. Ressalta-se que as repetições ocorreram tanto dentro da mesma base de dados, com o mesmo ou diferentes descritores, quanto em bases diferentes. Na sequência, os estudos foram selecionados para uma análise completa, de acordo com seu título, resumo e aderência ao tema proposto. Restou, assim, 21 estudos. Após a análise e leitura completa, 16 artigos foram selecionados para esta pesquisa, todos redigidos em língua inglesa, tendo sido seis $(37,5 \%)$ realizados nos Estados Unidos, três $(18,7 \%)$ na Alemanha, três $(18,7 \%)$ no Canadá, um $(6,2 \%)$ em Burundi, um (6,2\%) na Dinamarca, um $(6,2 \%)$ na Noruega e um $(6,2 \%)$ na Suíça.

Conforme apontado na metodologia, no Quadro 1 consta a síntese dos estudos selecionados para esta pesquisa, informando o autor/ano, país de realização do estudo, descritores utilizados para busca, objetivos, tipo de estudo e resultados alcançados que possibilitaram a análise e discussão do presente artigo.

Por meio do Quadro 2 e do Gráfico 1, é possível verificar os indicadores relacionados aos transtornos mentais em crianças e adolescentes, bem como a respectiva frequência. Foram identificados 20 indicadores, dos quais quatro destacaram-se: nível socioeconômico da família - renda familiar (seis), escolaridade dos pais (quatro), status de ocupação dos pais (dois) e privação social (dois).

O Quadro 2 possibilitou, ainda, o agrupamento dos textos a partir da associação dos fatores relacionados aos indicadores identificados, tendo sido identificados 17 fatores, dos quais quatro apresentaram maior frequência: baixa escolaridade dos pais (quatro), baixa renda familiar (três), gênero feminino (dois) e associação com a internalização dos sintomas (dois). A Tabela 1 apresenta a frequência dos fatores.

Com relação ao indicador "nível socioeconômico da família (renda familiar)", os estudos de Reiss (2013), Boe et al. (2011), McLaughlin et al. (2011), McLaughlin et al. (2012) e Anderson et al. (2012) apontam que a baixa renda familiar encontra-se relacionada a problemas de saúde mental na infância e na adolescência, porém os autores não descrevem de forma objetiva o porquê dessa relação. Reiss (2013), por exemplo, aponta que a privação de recursos materiais na infância estaria mais fortemente relacionada ao início do transtorno do que seu curso ou gravidade. Acrescenta que o estresse associado a uma baixa posição social contribui para o desenvolvimento de desordens de saúde mental. McLaughlin et al. (2011), por sua vez, revelam que percepções de status social relativamente baixo podem gerar comparações sociais que acabam proporcionando sentimentos de baixa autoestima entre crianças e adolescentes. No trabalho de Anderson et al. (2012), evidenciou-se que $25 \%$ dos pacientes residiam em áreas correspondentes ao pior estrato de privação material. 
Figura 1 - Apresentação do fluxo de seleção dos estudos que compuseram o corpus da pesquisa
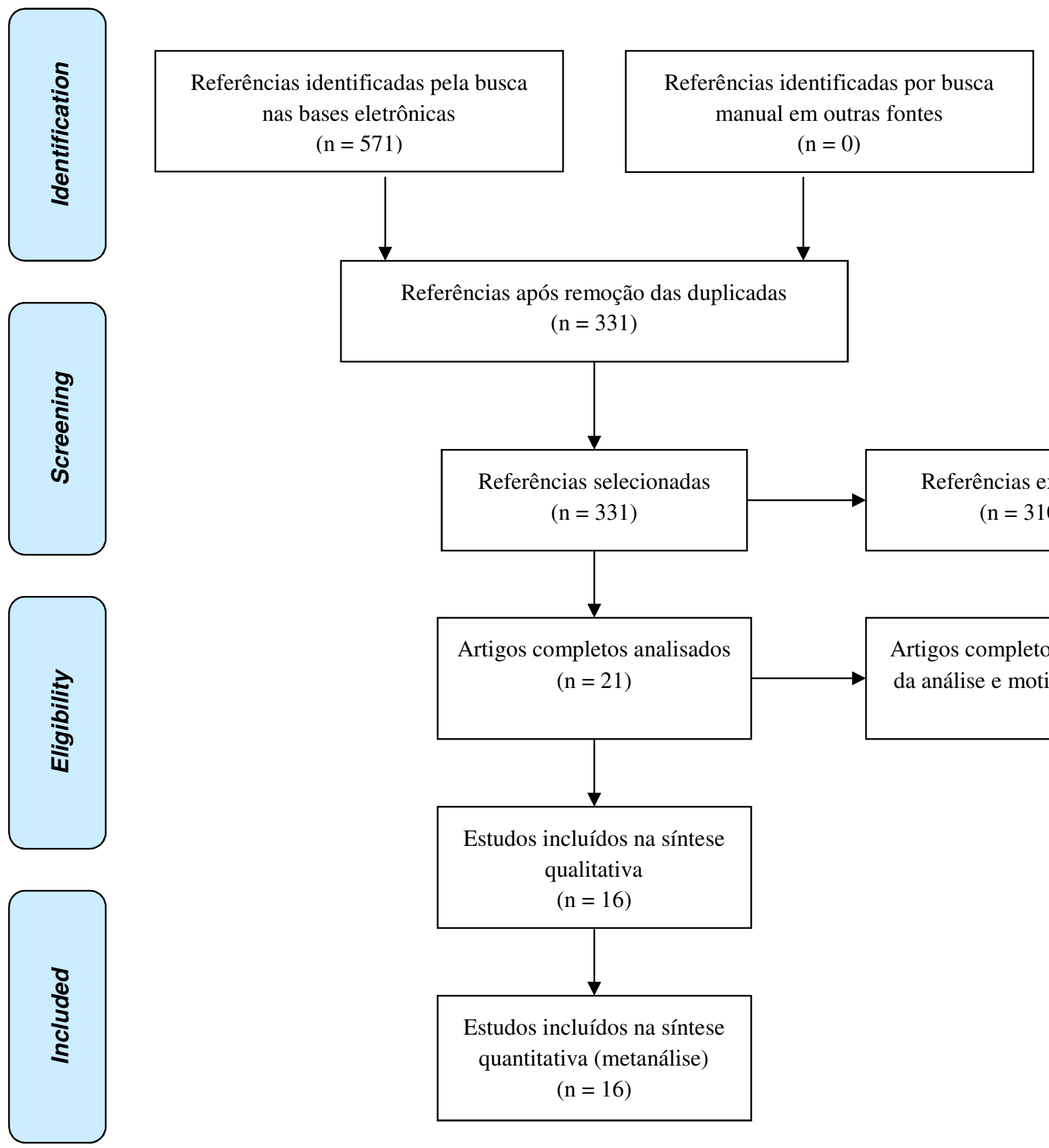

Referencias após renoção das duplicadas

$$
(\mathrm{n}=331)
$$
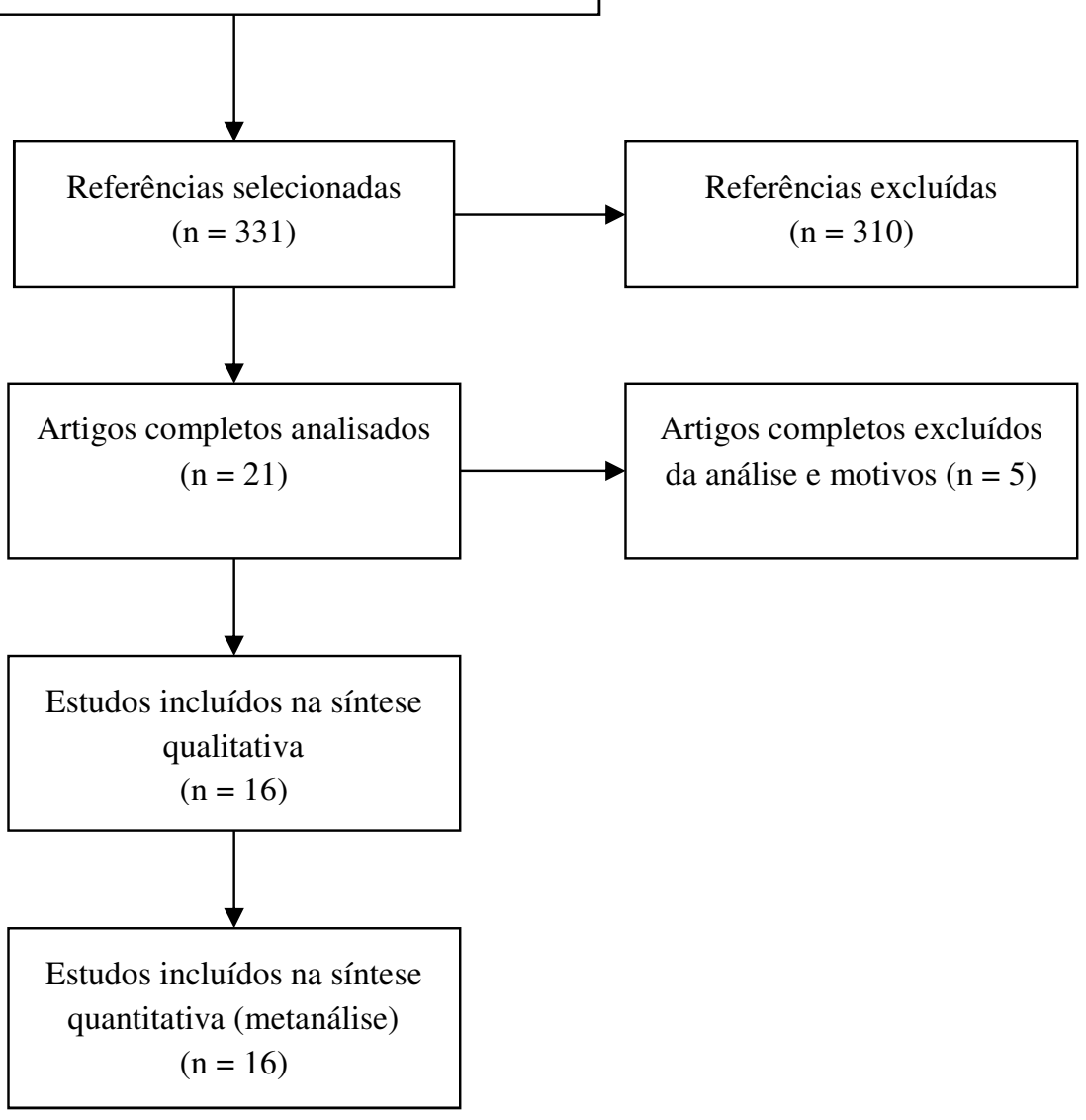

Fonte: Adaptado de Liberati et al. (2009). 
Quadro 1 - Síntese dos artigos selecionados.

continua

\begin{tabular}{|c|c|c|c|c|c|c|}
\hline & Autor/ano & País & Descritores & Objetivos & $\begin{array}{l}\text { Tipo de } \\
\text { estudo }\end{array}$ & Resultados \\
\hline I & Reiss (2013) & Alemanha & $\begin{array}{l}\text { Indicators AND } \\
\text { children with mental } \\
\text { disorder }+ \text { Indicators } \\
\text { AND adolescents with } \\
\text { mental disorder }\end{array}$ & $\begin{array}{l}\text { Investigar a definição e } \\
\text { a operacionalização do } \\
\text { status socioeconômico } \\
\text { (SES) e transtorno mental, } \\
\text { determinar a relação entre } \\
\text { um baixo SES e problemas } \\
\text { de saúde mental na infância } \\
\text { e adolescência, e discutir as } \\
\text { possíveis explicações para a } \\
\text { relação encontrada. }\end{array}$ & $\begin{array}{l}\text { Revisão } \\
\text { sistemática }\end{array}$ & $\begin{array}{l}\text { Renda familiar baixa e } \\
\text { escolaridade dos pais têm } \\
\text { grande efeito sobre a saúde } \\
\text { mental das crianças e } \\
\text { adolescentes. }\end{array}$ \\
\hline II & Hall et al. (2014) & Burundi & $\begin{array}{l}\text { Indicators AND } \\
\text { children with mental } \\
\text { disorder }\end{array}$ & $\begin{array}{l}\text { Examinar a associação } \\
\text { longitudinal entre o capital } \\
\text { social cognitivo e a saúde } \\
\text { mental (depressão e } \\
\text { sintomas de transtorno de } \\
\text { estresse pós-traumático) em } \\
\text { crianças em Burundi. }\end{array}$ & $\begin{array}{l}\text { Estudo } \\
\text { longitudinal }\end{array}$ & $\begin{array}{l}\text { O capital social cognitivo } \\
\text { está relacionado com a } \\
\text { deterioração da saúde } \\
\text { mental infantil e um } \\
\text { aumento no suporte social. }\end{array}$ \\
\hline III & $\begin{array}{l}\text { Suchert, } \\
\text { Hanewinkel e } \\
\text { Isensee (2015) }\end{array}$ & Alemanha & $\begin{array}{l}\text { Indicators AND } \\
\text { children with mental } \\
\text { disorder }+ \text { Indicators } \\
\text { AND adolescents with } \\
\text { mental disorder }\end{array}$ & $\begin{array}{l}\text { Realizar uma revisão } \\
\text { sistemática dos estudos que } \\
\text { abordam a relação entre } \\
\text { Comportamento Sedentário } \\
\text { (CS) e indicadores de } \\
\text { saúde mental em crianças e } \\
\text { adolescentes. }\end{array}$ & $\begin{array}{l}\text { Revisão } \\
\text { sistemática }\end{array}$ & $\begin{array}{l}\text { Há uma grande relação } \\
\text { entre CS e sintomas de } \\
\text { depressão. }\end{array}$ \\
\hline IV & Boe et al. (2011) & Noruega & $\begin{array}{l}\text { Indicators AND } \\
\text { children with mental } \\
\text { disorder }+ \text { Indicators } \\
\text { AND adolescents with } \\
\text { mental disorder }\end{array}$ & $\begin{array}{l}\text { Buscar a relação entre } \\
\text { problemas na saúde } \\
\text { mental de crianças e SES, } \\
\text { incluindo informações mais } \\
\text { detalhadas. }\end{array}$ & $\begin{array}{l}\text { Estudo } \\
\text { longitudinal }\end{array}$ & $\begin{array}{l}\text { A baixa escolaridade } \\
\text { dos pais está relacionada } \\
\text { com problemas de saúde } \\
\text { mental (principalmente } \\
\text { hiperatividade e } \\
\text { desatenção). Também foi } \\
\text { observado que a renda } \\
\text { familiar tem grande } \\
\text { influência em problemas } \\
\text { emocionais. }\end{array}$ \\
\hline V & $\begin{array}{l}\text { McLaughlin et } \\
\text { al. (2011) }\end{array}$ & $\begin{array}{l}\text { Estados } \\
\text { Unidos }\end{array}$ & $\begin{array}{l}\text { Indicators AND } \\
\text { children with mental } \\
\text { disorder }+ \text { Indicators } \\
\text { AND adolescents with } \\
\text { mental disorder }\end{array}$ & $\begin{array}{l}\text { Cobrir a lacuna da } \\
\text { literatura em relação a } \\
\text { estudos que envolvam } \\
\text { vários indicadores } \\
\text { socioeconômicos na } \\
\text { infância e o começo da } \\
\text { doença mental em adultos. }\end{array}$ & $\begin{array}{l}\text { Estudo } \\
\text { longitudinal }\end{array}$ & $\begin{array}{l}\text { Adversidades financeiras } \\
\text { na infância não têm relação } \\
\text { com a persistência e } \\
\text { severidade do transtorno. } \\
\text { Baixo nível de escolaridade } \\
\text { dos pais tem relação com a } \\
\text { persistência e severidade do } \\
\text { transtorno, mas não com o } \\
\text { começo da doença. }\end{array}$ \\
\hline VI & $\begin{array}{l}\text { Golembo-Smith } \\
\text { et al. (2012) }\end{array}$ & Dinamarca & $\begin{array}{l}\text { Indicators AND } \\
\text { children with mental } \\
\text { disorder }\end{array}$ & $\begin{array}{l}\text { Utilizar dados coletados } \\
\text { durante quase } 50 \text { anos } \\
\text { para medir se indicadores } \\
\text { de instabilidade no } \\
\text { neurodesenvolvimento } \\
\text { (habilidades cognitivas, } \\
\text { coordenação motora, } \\
\text { lateralidade = variáveis), } \\
\text { risco genético e } \\
\text { pequenas anormalidades } \\
\text { motoras podem prever a } \\
\text { esquizofrenia em adultos. }\end{array}$ & $\begin{array}{l}\text { Estudo } \\
\text { longitudinal }\end{array}$ & $\begin{array}{l}\text { Risco genético e pequenas } \\
\text { anormalidades motoras } \\
\text { são bons indicadores para } \\
\text { prever a esquizofrenia } \\
\text { adulta. }\end{array}$ \\
\hline
\end{tabular}


Quadro 1 - Síntese dos artigos selecionados.

continua

\begin{tabular}{|c|c|c|c|c|c|c|}
\hline & Autor/ano & País & Descritores & Objetivos & $\begin{array}{l}\text { Tipo de } \\
\text { estudo }\end{array}$ & Resultados \\
\hline VII & $\begin{array}{l}\text { Martelon et al. } \\
(2012)\end{array}$ & $\begin{array}{l}\text { Estados } \\
\text { Unidos }\end{array}$ & $\begin{array}{l}\text { Indicators AND } \\
\text { children with mental } \\
\text { disorder }+ \text { Indicators } \\
\text { AND adolescents with } \\
\text { mental disorder }\end{array}$ & $\begin{array}{l}\text { Examinar a associação entre } \\
\text { dificuldades obstétricas e } \\
\text { no período perinatal e o } \\
\text { transtorno bipolar infantil } \\
\text { (pediátrico). }\end{array}$ & $\begin{array}{l}\text { Estudo } \\
\text { longitudinal e } \\
\text { caso-controle } \\
\text { de família }\end{array}$ & $\begin{array}{l}\text { Não foi encontrada } \\
\text { nenhuma relação entre } \\
\text { dificuldades obstétricas } \\
\text { e perinatais e transtorno } \\
\text { bipolar infantil. As mães } \\
\text { de crianças com transtorno } \\
\text { bipolar reportaram ter } \\
\text { mais dificuldades durante } \\
\text { a infância do que as mães } \\
\text { com crianças saudáveis. }\end{array}$ \\
\hline VIII & $\begin{array}{l}\text { Coulombe et al. } \\
(2010)\end{array}$ & Canadá & $\begin{array}{l}\text { Indicators AND } \\
\text { children with mental } \\
\text { disorder }+ \text { Indicators } \\
\text { AND adolescents with } \\
\text { mental disorder }\end{array}$ & $\begin{array}{l}\text { Entender melhor a relação } \\
\text { entre problemas para } \\
\text { dormir, indicadores de sono } \\
\text { inadequado e sintomas de } \\
\text { psicopatologias em crianças } \\
\text { saudáveis de } 4-11 \text { anos. }\end{array}$ & $\begin{array}{l}\text { Estudo } \\
\text { longitudinal }\end{array}$ & $\begin{array}{l}\text { A relação entre problemas } \\
\text { para dormir, indicadores de } \\
\text { sono inadequado e sintomas } \\
\text { de psicopatologias é muito } \\
\text { complexa, pois há uma } \\
\text { confusão nas variáveis. }\end{array}$ \\
\hline IX & $\begin{array}{l}\text { Olfson et al. } \\
(2014)\end{array}$ & $\begin{array}{l}\text { Estados } \\
\text { Unidos }\end{array}$ & $\begin{array}{l}\text { Indicators AND } \\
\text { children with mental } \\
\text { disorder }+ \text { Indicators } \\
\text { AND adolescents with } \\
\text { mental disorder }\end{array}$ & $\begin{array}{l}\text { Acessar as tendências e } \\
\text { padrões nacionais (EUA) } \\
\text { de atendimento em saúde } \\
\text { mental em crianças, } \\
\text { adolescentes e adultos em } \\
\text { escritórios médicos. }\end{array}$ & $\begin{array}{l}\text { Estudo } \\
\text { longitudinal }\end{array}$ & $\begin{array}{l}\text { O cuidado com a saúde } \\
\text { mental infantil cresceu mais } \\
\text { rápido do que a adulta e } \\
\text { coincidiu com o aumento } \\
\text { do uso de medicação } \\
\text { psicotrópica. }\end{array}$ \\
\hline X & $\begin{array}{l}\text { Weitkamp et al. } \\
\text { (2013) }\end{array}$ & Alemanha & $\begin{array}{l}\text { Indicators AND } \\
\text { children with mental } \\
\text { disorder }+ \text { Indicators } \\
\text { AND adolescents with } \\
\text { mental disorder }\end{array}$ & $\begin{array}{l}\text { Identificar a relação } \\
\text { entre internalização e } \\
\text { externalização de patologias } \\
\text { e qualidade de vida, } \\
\text { relacionada à saúde, de } \\
\text { crianças e adolescentes, } \\
\text { como também os aspectos } \\
\text { das crianças e do ambiente } \\
\text { que afetam a qualidade de } \\
\text { vida. }\end{array}$ & $\begin{array}{l}\text { Estudo } \\
\text { longitudinal }\end{array}$ & $\begin{array}{l}\text { Tanto para os pais quanto } \\
\text { para as crianças, o bem- } \\
\text { estar físico e psicológico } \\
\text { influencia muito a } \\
\text { qualidade de vida, que } \\
\text { tem relação mais forte } \\
\text { com a internalização de } \\
\text { patologia do que com a } \\
\text { externalização. }\end{array}$ \\
\hline XI & $\begin{array}{l}\text { McLaughlin et } \\
\text { al. (2012) }\end{array}$ & $\begin{array}{l}\text { Estados } \\
\text { Unidos }\end{array}$ & $\begin{array}{l}\text { Indicators AND } \\
\text { adolescents with } \\
\text { mental disorder }\end{array}$ & $\begin{array}{l}\text { Determinar quais aspectos } \\
\text { do SES estão relacionados } \\
\text { com a doença mental. }\end{array}$ & $\begin{array}{l}\text { Estudo } \\
\text { longitudinal }\end{array}$ & $\begin{array}{l}\text { Escolaridade parental e } \\
\text { status social subjetivo } \\
\text { foram relacionados com } \\
\text { transtorno de ansiedade. } \\
\text { Transtornos de humor } \\
\text { foram relacionados com } \\
\text { privação relativa e status } \\
\text { social subjetivo. Status } \\
\text { social subjetivo foi o único } \\
\text { indicador relacionado } \\
\text { com transtorno de } \\
\text { comportamento disruptivo e } \\
\text { uso de substâncias. }\end{array}$ \\
\hline XII & $\begin{array}{l}\text { Anderson et al. } \\
\text { (2012) }\end{array}$ & Canadá & $\begin{array}{l}\text { Indicators AND } \\
\text { adolescents with } \\
\text { mental disorder }\end{array}$ & $\begin{array}{l}\text { Obter uma estimativa da } \\
\text { incidência dos primeiros } \\
\text { episódios do espectro de } \\
\text { esquizofrenia psicótica } \\
\text { em adolescentes e jovens } \\
\text { adultos utilizando dados do } \\
\text { serviço social e de saúde } \\
\text { canadense. }\end{array}$ & $\begin{array}{l}\text { Estudo de } \\
\text { caso }\end{array}$ & $\begin{array}{l}\text { O índice de espectro de } \\
\text { esquizofrenia psicótica } \\
\text { foi mais alto em pessoas } \\
\text { vivendo em áreas com } \\
\text { maior privação material do } \\
\text { que em áreas com menor. }\end{array}$ \\
\hline
\end{tabular}


Quadro 1 - Síntese dos artigos selecionados.

\begin{tabular}{|c|c|c|c|c|c|c|}
\hline & Autor/ano & País & Descritores & Objetivos & $\begin{array}{l}\text { Tipo de } \\
\text { estudo }\end{array}$ & Resultados \\
\hline XIII & $\begin{array}{l}\text { Tackett et al. } \\
\text { (2014) }\end{array}$ & Canadá & $\begin{array}{l}\text { Indicators AND } \\
\text { children with mental } \\
\text { disorder }+ \text { Indicators } \\
\text { AND adolescents with } \\
\text { mental disorder }\end{array}$ & $\begin{array}{l}\text { Estender as pesquisas } \\
\text { sobre a hipótese do "duplo } \\
\text { hormônio" (que afirma } \\
\text { que a testosterona e o } \\
\text { cortisol, juntos, regulam } \\
\text { a agressividade e o } \\
\text { comportamento dominante } \\
\text { em crianças e adultos), } \\
\text { testando a interação entre } \\
\text { testosterona, cortisol e } \\
\text { traços de transtornos de } \\
\text { personalidade. }\end{array}$ & $\begin{array}{l}\text { Estudo } \\
\text { longitudinal }\end{array}$ & $\begin{array}{l}\text { Altos níveis de testosterona } \\
\text { foram associados } \\
\text { com problemas de } \\
\text { externalização, mas } \\
\text { somente quando o nível de } \\
\text { cortisol era baixo. }\end{array}$ \\
\hline XIV & $\begin{array}{l}\text { Zima et al. } \\
(2010)\end{array}$ & $\begin{array}{l}\text { Estados } \\
\text { Unidos }\end{array}$ & $\begin{array}{l}\text { Indicators AND } \\
\text { children with } \\
\text { mental disorder }+ \\
\text { Indicadores E crianças } \\
\text { com transtorno mental }\end{array}$ & $\begin{array}{l}\text { Examinar se as crianças } \\
\text { que possuem Transtorno } \\
\text { do Déficit de Atenção com } \\
\text { Hiperatividade (TDAH) e } \\
\text { são tratadas em clínicas de } \\
\text { saúde mental especializadas } \\
\text { possuem casos mais graves } \\
\text { do que as que são tratadas } \\
\text { na atenção primária. }\end{array}$ & $\begin{array}{l}\text { Estudo } \\
\text { de corte } \\
\text { longitudinal }\end{array}$ & $\begin{array}{l}\text { A gravidade dos casos } \\
\text { clínicos não difere caso a } \\
\text { criança seja tratada em uma } \\
\text { unidade primária ou em } \\
\text { uma clínica especializada. }\end{array}$ \\
\hline XV & $\begin{array}{l}\text { Allen et al. } \\
(2010)\end{array}$ & Suíça & $\begin{array}{l}\text { Indicators AND } \\
\text { children with mental } \\
\text { disorder }\end{array}$ & $\begin{array}{l}\text { Analisar a frequência dos } \\
\text { critérios de sintomas e } \\
\text { diagnósticos do DSM-IV de } \\
\text { transtorno de ansiedade de } \\
\text { separação. }\end{array}$ & $\begin{array}{l}\text { Estudo } \\
\text { longitudinal }\end{array}$ & $\begin{array}{l}\text { Não houve nenhuma } \\
\text { diferença entre as idades } \\
\text { e sexo das crianças, mas } \\
\text { maior relutância a ir para a } \\
\text { escola em meninas do que } \\
\text { em meninos. }\end{array}$ \\
\hline XVI & $\begin{array}{l}\text { Bitsko et al. } \\
(2014)\end{array}$ & $\begin{array}{l}\text { Estados } \\
\text { Unidos }\end{array}$ & $\begin{array}{l}\text { Indicators AND } \\
\text { children with mental } \\
\text { disorder }\end{array}$ & $\begin{array}{l}\text { Fornecer estimativas } \\
\text { recentes da prevalência } \\
\text { da síndrome de Tourette e } \\
\text { descrever a associação entre } \\
\text { a síndrome e indicadores de } \\
\text { saúde e funcionamento. }\end{array}$ & $\begin{array}{l}\text { Estudo } \\
\text { longitudinal }\end{array}$ & $\begin{array}{l}\text { Comparando com crianças } \\
\text { saudáveis, as crianças } \\
\text { com síndrome de Tourette } \\
\text { tinham mais chance de } \\
\text { possuir outras doenças } \\
\text { neurológicas. }\end{array}$ \\
\hline
\end{tabular}

Quadro 2 - Indicadores e fatores associados identificados na pesquisa

\begin{tabular}{|c|c|c|c|}
\hline & Autor/ano & Indicadores & Fatores associados \\
\hline I & Reiss (2013) & $\begin{array}{l}\text { Renda familiar. } \\
\text { Escolaridade dos pais. } \\
\text { Status de ocupação dos pais. } \\
\text { Nível socioeconômico da família. }\end{array}$ & $\begin{array}{l}\text { Baixa renda familiar. } \\
\text { Baixa escolaridade dos pais. } \\
\text { Crianças pequenas: maior incidência relacionada à desigualdade } \\
\text { econômica. }\end{array}$ \\
\hline II & Hall et al. (2014) & Capital social cognitivo. & Coesão e reciprocidade dentro da comunidade. \\
\hline III & $\begin{array}{l}\text { Suchert, } \\
\text { Hanewinkel e } \\
\text { Isensee (2015) }\end{array}$ & $\mathrm{CS}$. & $\begin{array}{l}\text { Gênero feminino. } \\
\text { Internalização de problemas. } \\
\text { Qualidade de vida e bem-estar. }\end{array}$ \\
\hline IV & Boe et al. (2011) & $\begin{array}{l}\text { Renda familiar. } \\
\text { Escolaridade dos pais. }\end{array}$ & $\begin{array}{l}\text { Baixa renda familiar. } \\
\text { Baixa escolaridade dos pais. }\end{array}$ \\
\hline V & $\begin{array}{l}\text { McLaughlin et } \\
\text { al. (2011) }\end{array}$ & $\begin{array}{l}\text { Nível socioeconômico da família. } \\
\text { Escolaridade dos pais. } \\
\text { Status de ocupação dos pais. }\end{array}$ & $\begin{array}{l}\text { Baixa escolaridade dos pais. } \\
\text { Status de ocupação dos pais baixo. }\end{array}$ \\
\hline
\end{tabular}


Quadro 2 - Indicadores e fatores associados identificados na pesquisa

conclusão

\begin{tabular}{|c|c|c|c|}
\hline & Autor/ano & Indicadores & Fatores associados \\
\hline VI & $\begin{array}{l}\text { Golembo-Smith } \\
\text { et al. (2012) }\end{array}$ & $\begin{array}{l}\text { Instabilidade no neurodesenvolvimento. } \\
\text { Risco genético. } \\
\text { Anormalidades motoras. }\end{array}$ & $\begin{array}{l}\text { Pai com diagnóstico de esquizofrenia. } \\
\text { Anormalidades motoras. }\end{array}$ \\
\hline VII & $\begin{array}{l}\text { Martelon et al. } \\
(2012)\end{array}$ & $\begin{array}{l}\text { Dificuldades obstétricas e no período } \\
\text { perinatal. }\end{array}$ & Sem relação direta com o transtorno bipolar. \\
\hline VIII & $\begin{array}{l}\text { Coulombe et al. } \\
(2010)\end{array}$ & Sono inadequado. & $\begin{array}{l}\text { Dificuldade no estabelecimento de fatores pela complexidade das } \\
\text { variáveis. }\end{array}$ \\
\hline IX & $\begin{array}{l}\text { Olfson et al. } \\
(2014)\end{array}$ & $\begin{array}{l}\text { Diagnóstico de transtorno mental. } \\
\text { Prescrição de medicamentos } \\
\text { psiquiátricos. } \\
\text { Recomendação para atendimento de } \\
\text { psicólogos e psiquiatras. }\end{array}$ & Número de consultas realizadas. \\
\hline $\mathrm{X}$ & $\begin{array}{l}\text { Weitkamp et al. } \\
\text { (2013) }\end{array}$ & Qualidade de vida. & Associação com a internalização dos sintomas. \\
\hline $\mathrm{XI}$ & $\begin{array}{l}\text { McLaughlin et } \\
\text { al. (2012) }\end{array}$ & $\begin{array}{l}\text { Renda familiar. } \\
\text { Escolaridade dos pais. } \\
\text { Privação social. } \\
\text { Nível socioeconômico da família. }\end{array}$ & $\begin{array}{l}\text { Baixa escolaridade dos pais. } \\
\text { Status social associado ao uso de substâncias. }\end{array}$ \\
\hline XII & $\begin{array}{l}\text { Anderson et al. } \\
(2012)\end{array}$ & $\begin{array}{l}\text { Privação social. } \\
\text { Privação material. }\end{array}$ & $\begin{array}{l}\text { Alto índice em pessoas vivendo em áreas com maior privação } \\
\text { material. }\end{array}$ \\
\hline XIII & $\begin{array}{l}\text { Tackett et al. } \\
(2014)\end{array}$ & Duplo hormônio (testosterona e cortisol). & Altos níveis de testosterona $\mathrm{x}$ baixos níveis de cortisol. \\
\hline XIV & $\begin{array}{l}\text { Zima et al. } \\
(2010)\end{array}$ & $\begin{array}{l}\text { Atendimento em clínicas especializadas e } \\
\text { na atenção primária. }\end{array}$ & Sem relação direta com a gravidade do transtorno. \\
\hline $\mathrm{XV}$ & $\begin{array}{l}\text { Allen et al. } \\
(2010)\end{array}$ & Critérios de sintomas DSM-IV. & Gênero feminino. \\
\hline XVI & $\begin{array}{l}\text { Bitsko et al. } \\
(2014)\end{array}$ & Síndrome de Tourette. & Maior probabilidade de incidência de doenças em crianças. \\
\hline
\end{tabular}

Gráfico 1 - Frequência absoluta dos indicadores identificados na pesquisa.

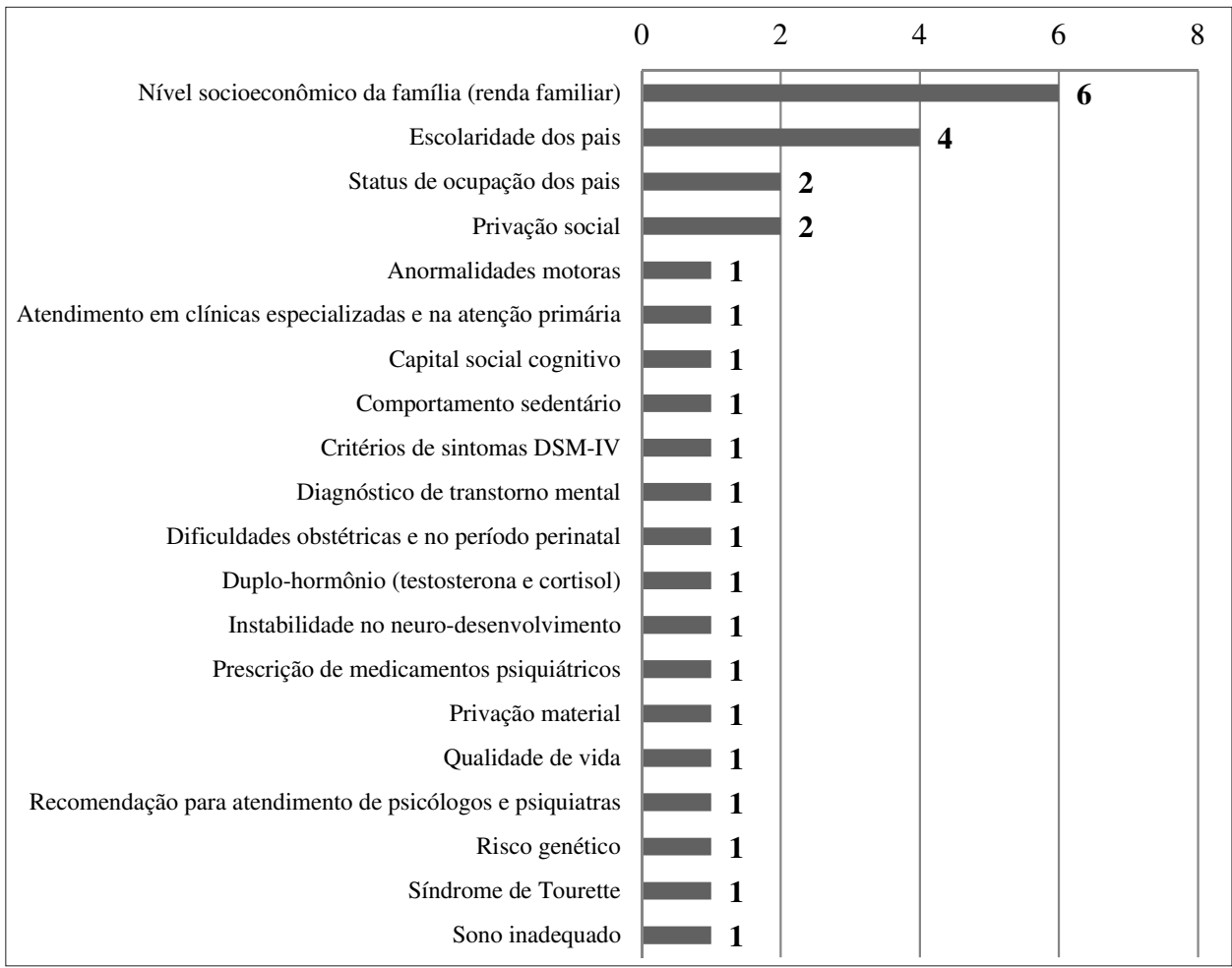


UMA REVISÃO INTEGRATIVA

Tabela 1 - Frequência absoluta e relativa dos fatores identificados na pesquisa

\begin{tabular}{l|c|c}
\hline \multicolumn{1}{c|}{ Fator associado } & FA & FR (\%) \\
\hline Baixa escolaridade dos pais & 4 & 16,7 \\
\hline Baixa renda familiar & 3 & 12,5 \\
\hline Gênero feminino & 2 & 8,3 \\
\hline Associação com a internalização dos sintomas & 2 & 8,3 \\
\hline Altos níveis de testosterona x baixos níveis de cortisol & 1 & 4,2 \\
\hline Anormalidades motoras & 1 & 4,2 \\
\hline Coesão e reciprocidade dentro da comunidade & 1 & 4,2 \\
\hline Crianças pequenas: maior incidência relacionada à desigualdade econômica & 1 & 4,2 \\
\hline Dificuldade no estabelecimento de fatores pela complexidade das variáveis & 1 & 4,2 \\
\hline Maior probabilidade de incidência de doenças em crianças & 1 & 4,2 \\
\hline Número de consultas realizadas & 1 & 4,2 \\
\hline Qualidade de vida e bem-estar & 1 & 4,2 \\
\hline Pai com diagnóstico de esquizofrenia & 1 & 4,2 \\
\hline Sem relação direta com a gravidade do transtorno & 1 & 4,2 \\
\hline Sem relação direta com o transtorno bipolar & 1 & 4,2 \\
\hline Status de ocupação dos pais baixo & 1 & 4,2 \\
\hline Status social associado ao uso de substâncias & 1 & 4,2 \\
\hline Total & $\mathbf{2 4}$ & $\mathbf{1 0 0 , 0}$ \\
\hline
\end{tabular}

No tocante à escolaridade dos pais, Reiss (2013), Boe et al. (2011), McLaughlin et al. (2011) e McLaughlin et al. (2012) afirmam que sua instrução está associada a quadros de transtornos mentais em crianças e adolescentes. Os resultados do estudo de Reiss (2013) sugerem que a educação dos pais em níveis elevados pode estar ligada a um melhor acesso a recursos, como tratamento de saúde mental. Compartilhando da mesma perspectiva, McLaughlin et al. (2011) salientam que o baixo nível de escolaridade dos pais reflete em uma desvantagem persistente na posição social, que é um marcador do acesso a recursos, ao conhecimento e às estruturas sociais, que promovem a saúde e bem-estar do ser humano.

O status de ocupação profissional dos pais também foi um dos indicadores que se evidenciaram nos estudos. Profissões apontadas como baixas (pouco prestígio social) nos estudos de McLaughlin et al. (2011) e Reiss (2013) estariam mais propensas ao desenvolvimento de problemas de saúde mental na infância e na adolescência. McLaughlin et al. (2011) apontam que a ocupação dos pais está associada ao prestígio e exposição diferencial a duras condições de trabalho, que poderiam ter repercussões nos níveis de estresse e são presenciados por crianças em seus lares.
A privação social nos primeiros anos de vida foi demonstrada nos estudos de McLaughlin et al. (2012) e Anderson et al. (2012) como um indicador de patologias mentais na infância, estando o fator relacionado ao status social da família, quando esta está envolvida com o uso de substâncias (MCLAUGHLIN et al., 2012) e possui um índice elevado de pessoas convivendo em um ambiente com privação material (ANDERSON et al., 2012).

Não menos importantes, como apontado no Gráfico 1, outros indicadores foram identificados e sua associação pode ser acompanhada no Quadro 2, sendo sumarizados a seguir.

O estudo realizado por Suchert, Hanewinkel e Isensee (2015) aponta que há uma diferença quando se trata dos gêneros: meninas que assistem a muita TV reportaram altos índices de sintomas depressivos, enquanto os meninos na mesma condição reportaram baixos índices de sintomas depressivos. Outros estudos provaram o contrário; então, não foi encontrado um padrão consistente entre o CS e a depressão; o mesmo aconteceu com os estudos que relacionam CS e ansiedade. Sete estudos analisaram a relação entre CS e internalização de problemas, concluindo-se que há relação direta entre os dois. Os estudos abordando bem-estar e qualidade de vida e CS provam haver relação 
indireta entre eles (SUCHERT; HANEWINKEL; ISENSEE, 2015).

Golembo-Smith et al. (2012) indicam que o risco genético e pequenas anormalidades motoras são bons indicadores para prever a esquizofrenia adulta. Já Martelon et al. (2012) examinaram a associação entre dificuldades obstétricas no período perinatal e o transtorno bipolar infantil (pediátrico). Nesse sentido, verificou-se que complicações obstétricas podem dificultar o desenvolvimento cerebral no período pré-natal e perinatal. Como resultado, as mães com crianças com tal transtorno reportaram ter mais dificuldades durante a infância do que as mães com crianças saudáveis.

Na pesquisa de Coulombe et al. (2010), a relação entre problemas para dormir, indicadores de sono inadequado e sintomas de psicopatologias foi caracterizada como muito complexa. Pesadelos e dificuldades para dormir, por exemplo, foram relacionados com humor ansioso/deprimido. Contudo, os autores alertam para uma confusão nas variáveis do sono, sendo necessária sua análise separadamente, para que a relação (indicadores do sono e psicopatologias) possa ser melhor entendida, haja vista que nem todas as variáveis estão significativamente associadas com todos os tipos de psicopatologia.

O estudo de Olfson et al. (2014) definiu quatro indicadores: diagnóstico de transtorno mental, prescrição para medicamento psicotrópico, recomendação para um psicoterapeuta e consultas com um psiquiatra. Como resultado, concluiu-se que: o número de consultas que resultaram em diagnóstico e recomendação dobrou para os jovens, mas não aumentou para os adultos; o cuidado com a saúde mental infantil cresceu em maior escala que a adulta e coincidiu com o aumento do uso de medicação psicotrópica; a grande maioria dos atendimentos em saúde mental em consultório é realizada por médicos que não são psiquiatras; o número de consultas que resultaram em prescrição aumentou tanto em adultos quanto em crianças.

Weitkamp et al. (2013) almejavam identificar a relação entre a internalização e externalização de sintomas e a qualidade de vida relacionada à saúde de crianças e adolescentes. Verificou-se que, tanto para os pais quanto para as crianças, o bem-estar físico e psicológico influenciou a qualidade de vida, que tem uma relação mais forte com a internalização dos sintomas do que com a externalização. Ainda, a internalização da patologia foi relacionada com a qualidade de vida diminuída em termos de bem-estar psicológico, apoio social e relação com os pares, bem como o bem-estar com o ambiente escolar (em moderado e grande efeito). Já a externalização da patologia esteve associada a baixos níveis de qualidade nas relações parentais e na autonomia.

O trabalho de Anderson et al. (2012) evidenciou que o índice de esquizofrenia foi mais alto em pessoas vivendo em áreas com maior privação material (mais pobres) do que em áreas com menores privações materiais. Como os dados da pesquisa foram advindos apenas de registros administrativos do sistema de saúde, possivelmente a causa da esquizofrenia poderia ser explicada por variáveis que não estavam disponíveis no banco de dados, como etnia e status de migração (ANDERSON et al., 2012).

A pesquisa de Tackett et al. (2014) visou a estender as pesquisas sobre a hipótese do "duplo hormônio" (que afirma que a testosterona e o cortisol, juntos, regulam a agressividade e comportamento dominante em crianças e adultos), testando a interação entre testosterona, cortisol e traços de transtornos de personalidade. Os resultados evidenciaram que altos níveis de testosterona foram associados com resultados de externalização dos sintomas, mas somente quando o nível de cortisol era baixo.

Zima et al. (2010) queriam examinar se as crianças que possuem TDAH e são tratadas em clínicas de saúde mental especializadas possuem quadros mais severos do que as que são tratadas na atenção primária. O estudo concluiu que a gravidade dos casos clínicos não se diferencia caso a criança seja tratada em uma unidade primária ou em uma clínica especializada.

$\mathrm{O}$ artigo de Allen et al. (2010) visou a analisar a frequência dos critérios de sintomas e diagnósticos do DSM-IV de transtorno de ansiedade de separação. Não houve nenhuma diferença entre as idades e sexo das crianças, mas uma maior relutância na frequência escolar em meninas. Já Bitsko et al. (2014) queriam analisar a relação da síndrome de Tourette com outras doenças neurológicas e a forma como afetavam a relação da criança com a família, a educação e a saúde. Comparando com as crianças saudáveis, as crianças com síndrome tinham maior probabilidade para o desenvolvimento de outros problemas. 


\section{DISCUSSÃO}

O SES, associado à baixa renda familiar, foi evidenciado nesta revisão como um dos indicadores de transtornos mentais na infância e na adolescência, vindo a corroborar outros estudos internacionais e nacionais, como apontado na revisão realizada por Matsukura et al. (2014). Contudo, os autores não descrevem de forma objetiva o porquê de a baixa renda familiar estar relacionada a problemas de saúde mental. Alguns apontamentos sugerem a privação de recursos materiais na infância, estando ela relacionada ao início do transtorno e não à sua gravidade, como também situações de estresse associadas a uma baixa posição social (REISS, 2013). Os resultados do estudo de Reiss (2013) também apontam que a renda familiar e a baixa escolaridade dos pais têm mais efeito sobre a saúde mental das crianças e adolescentes do que o desemprego dos pais e/ou status profissional baixo. Ainda, os problemas emocionais na infância estão relacionados à baixa renda familiar, como informado por Boe et al. (2011).

A escolaridade dos pais foi o segundo indicador identificado nesta pesquisa como associado a patologias psiquiátricas. De fato, verificou-se que muitas vezes a baixa renda familiar e a baixa escolaridade dos genitores podem indicar a presença de problemas mentais na infância e na adolescência, como os transtornos de ansiedade, analisados no estudo de McLaughlin et al. (2012). Tal evidência também é apontada por Matsukura et al. (2014), que disserta que a associação entre esses dois fatores encontra-se diretamente relacionada a problemas comportamentais de crianças.

O mesmo indicador indicou um alto nível de sintomas relacionados a problemas de saúde mental, principalmente hiperatividade e desatenção, e, quando isolado, alto nível de problemas emocionais. Já quando associado à escolaridade parental e à renda familiar, esses fatores influenciaram a hiperatividade, desatenção e problemas de conduta (BOE et al., 2011). Ainda, a baixa escolaridade dos genitores esteve associada à severidade do transtorno em crianças e adolescentes, sendo que, quanto mais baixa a escolaridade, maior a gravidade do quadro (MCLAUGHLIN et al., 2011).

Por fim, as questões de gênero, sobretudo quando associadas ao sexo feminino e à baixa participação da família frente às atividades acadêmicas da criança ou do adolescente, foram relatadas como potenciais fatores associados a quadros de transtornos mentais nessa fase do desenvolvimento (AVANCI et al., 2007).

\section{CONCLUSÃO}

Por meio deste estudo, identificaram-se alguns fatores associados a casos de transtornos mentais na infância e adolescência, corroborando publicações anteriores.

A baixa renda familiar e a baixa escolaridade dos pais evidenciaram-se como principais problemas relacionados à saúde mental, de acordo com a literatura pesquisada, o que contrapõe à garantia do direito fundamental à vida e à saúde, pressuposto do Estatuto da Criança e do Adolescente (ECA). Tais fatores devem ser considerados em propostas de programas de promoção, prevenção e tratamento, visando à implementação de políticas públicas voltadas à garantia de direitos de crianças e adolescentes.

Por outro lado, esta pesquisa identificou a existência de uma gama de variáveis, tais como: altos níveis de testosterona, baixos níveis de cortisol, anormalidades motoras, coesão e reciprocidade dentro da comunidade, idade, quantidade de consultas realizadas, qualidade de vida, pais com diagnóstico de transtornos mentais, status de ocupação dos genitores e uso de substâncias, que estão relacionadas ao tema, necessitando, assim, ser foco de estudos, a fim de possibilitar novos olhares e perspectivas frente a esse público.

\section{REFERÊNCIAS}

ALLEN, J. L., LAVALLEE, K. L., HERREN, C., RUHE, K., SCHNEIDER, S. DSM-IV criteria for childhood separation anxiety disorder: informant, age, and sex differences. Journal of Anxiety Disorders, v. 24, p. 946952, 2010. Disponível em: http://www.ncbi.nlm.nih.gov/ pubmed/20675099 Acesso em: 10 set. 2015.

ANDERSON, K. K., FUHRER, R., ABRAHAMOWICZ, M., MALLA, A. K. The Incidence of First-Episode Schizophrenia-Spectrum Psychosis in Adolescents and Young Adults in Montreal: An Estimate From an Administrative Claims Database. Canadian Journal of Psychiatry, v. 57, n. 10, p. 626-633, 2012. Disponível em: http://www.ncbi.nlm.nih.gov/pubmed/23072954. Acesso em: 10 set. 2015.

AVANCI, J. Q., ASSIS, S.G., OLIVEIRA, R.V.C., FERREIRA, R.M., PESCE, R.P.: Fatores associados aos problemas de saúde mental em adolescentes. Psicologia: Teoria e Pesquisa, v. 23, n. 3, p. 287-294, 2007. 
BITSKO, R. H., HOLBROOK, J. R., VISSER, S. N., MINK, J. W., ZINNER, S. H., GHANDOUR, R. M., BLUMBERG, S. J. A National Profile of Tourette Syndrome, 2011-2012. Journal of developmental and behavioral paediatrics, v. 35, n. 5, p. 317-322, 2014. Disponível em: http://www.ncbi. nlm.nih.gov/pubmed/24906033. Acesso em: 10 set. 2015.

BOE, T., OVERLAND, S., LUNDERVOLD, A. J., HYSING, M. Socioeconomic status and children's mental health: results from the Bergen Child Study. Social Psychiatry and Psychiatric Epidemiology, v. 47, p. 1557 1566, Dezembro, 2011. Disponível em: http://link.springer. com/article/10.1007\%2Fs00127-011-0462-9. Acesso em: 2 set. 2015 .

CARVALHO, P. D. D., BARROS, M. V. G., SANTOS, C. M., MELO, E. N., OLIVEIRA, N. K. R., LIMA, R. A. Prevalência e fatores associados a indicadores negativos de saúde mental em adolescentes estudantes do ensino médio em Pernambuco, Brasil. Revista Brasileira de Saúde Materno Infantil, v. 11, n. 3, 2011. Disponível em: $<$ http:// www.scielo.br/scielo.php?script=sci_arttext\&pid $=\mathrm{S} 1519$ $38292011000300003 \& \operatorname{lng}=\mathrm{en} \& n r m=\mathrm{iso}>$. Acesso em: 15 fev. 2016.

COULOMBE, J. A., REID, G. J., BOYLE, M. H., RACINE, Y. Concurrent Associations among Sleep Problems, Indicators of Inadequate Sleep, Psychopathology, and Shared Risk Factors in a Population-based Sample of Healthy Ontario Children. Journal of Pediatric Psychology, v. 7, p. 790-799, 2010. Disponível em: http://www.ncbi.nlm.nih. gov/pubmed/19923203 Acesso em: 2 set. 2015.

COUTO, M. C. V., DUARTE, C. S., DELGADO, P. G. G. A saúde mental infantil na Saúde Pública brasileira: situação atual e desafios. Revista Brasileira Psiquiatria, v. 30, n. 4, p. 390-8, 2008.

FATORI DE SÁ, D. G., BORDIN, I. A. S., MARTIN, C. S. P. D. Fatores de risco para problemas de saúde mental na infância/adolescência. Psicologia: Teoria e Pesquisa, v. 26, n. 4, p. 643-652, 2010.

GOLEMBO-SMITH, S., SCHIFFMAN, J., KLINE, E., SORENSEN, H. J., MORTENSEN, E. L., STAPLETON, L., HAYASHI, K., MICHELSEN, N. M., EKSTROM, M., MEDNICK, S. Premorbid multivariate markers of neurodevelopmental instability in the prediction of adult schizophrenia-spectrum disorder: a high-risk prospective investigation. Elsevier: Schizophrenia Research, v. 139, p. 129-135, Junho, 2012. Disponível em: <http://www.ncbi. nlm.nih.gov/pmc/articles/PMC3393829/> Acesso: 2 set. 2015.

HALL, B. J., WIETSE, T. A., JORDANS, M. J. D., BASS, J., DE JONG, J. T. V. M. Understanding resilience in armed conflict: Social resources and mental health of children in Burundi. Elsevier: Social Science \& Medicine, v. 114, p. 121-128, Fevereiro, 2014. Disponível em:
$<$ http://www.sciencedirect.com/science/article/pii/ S0277953614003360>. Acesso em: 2 set. 2015.

LIBERATI, A., ALTMAN, D., TETZLAFF, J., MULROW, C., GOTZSCHE, P. C., IOANNIDIS, J. P. A., CLARKE, M., DEVEREAUX, P. J., KLEIJNEN, J., MOHER, D. The PRISMA statement for reporting systematic reviews and meta-analyses of studies that evaluate health care interventions: explanation and elaboration. Annals of Internal Medicine, 151: 65-94, 2009. Disponível em:

$<$ http://citeseerx.ist.psu.edu/viewdoc/download;jsessionid= 88C5D5BABCA701D766CD30434D6D2578?doi=10.1.1. 689.2699\&rep=rep1\&type=pdf $>$ Acesso em: 23 set. 2016.

MARTELON, M., WILENS, T. E., ANDERSON, J. P., MORRISON, N. R., WOZNIAK J. Are obstetrical, perinatal, and infantile difficulties associated with pediatric bipolar disorder? Bipolar Disorders - An International Journal of Psychiatry and Neurosciences, v. 14, n. 14, p. 507-514, 2012. Disponível em: <http://www.ncbi.nlm.nih. gov/pubmed/22642419> Acesso em: 2 set. 2015.

MATSUKURA, T. S., FERNANDES, A. D. S. A., CID, M. F. B.: Saúde mental infantil em contextos de desvantagem socioeconômica: fatores de risco e proteção. Cadernos de Terapia Ocupacional da UFSCar, v. 22, n. 2, 2014.

MCLAUGHLIN, K. A., COSTELLO, E. J., LEBLANC, W., SAMPSON, N. A., KESSLER, R. C. Socioeconomic Status and Adolescent Mental Disorders. American Journal of Public Health, v. 102, n. 9, p. 1742-1750, 2012. Disponível em: $\quad<$ http://www.ncbi.nlm.nih.gov/pubmed/22873479>. Acesso em: 2 set. 2015.

MCLAUGHLIN, K. A., BRESLAU, J., GREEN, J. G., LAKOMA, M. D., SAMPSON, N. A., ZASLAVSKY, A. M., KESSLER, R. C.: Childhood socio-economic status and the onset, persistence, and severity of DSM-IV mental disorders in a US national sample. Elsevier: Social Science \& Medicine, v. 73, p. 1088 - 1096, Julho, 2011. Disponível em: $\quad<$ http://www.sciencedirect.com/science/article/pii/ S0277953611003571> Acesso em: 2 set. 2015.

MENDES, K. D. S., SILVEIRA, R. C. C. P., GALVÃO, C. M. Revisão integrativa: método de pesquisa para a incorporação de evidências na saúde e na enfermagem. Texto \& Contexto-Enfermagem, v. 17, n. 4, p. 758-764, 2008.

OLFSON, M., BLANCO, C., WANG, S., LAJE, G., CORRELL, C. U. National Trends in the Mental Health Care of Children, Adolescents, and Adults by Office-Based Physicians. JAMA Psychiatry, v. 71, n. 1, p. 81-90, 2014. Disponível em: http://archpsyc.jamanetwork.com/article. aspx?articleid=1784344 Acesso em: 9 de out. 2015.

REISS, F.: Socioeconomic inequalities and mental health problems in children and adolescents. Elsevier: Social Science \& Medicine, v. 90, p. 24-31, Maio 2013. Disponível 
em: $\quad<\mathrm{http}: / /$ www.sciencedirect.com/science/article/pii/ S0277953613002608>. Acesso em: 2 set. 2015.

SUCHERT, V., HANEWINKEL, R., ISENSEE, B. Sedentary behavior and indicators of mental health in school-aged children and adolescents: A systematic review. Elsevier: Preventive Medicine, v. 76, p. 48-57, Abril, 2015. Disponível em: <http://www.researchgate. net/publication/275218836_Sedentary_behavior_and indicators_of_mental_health_in_school-aged_children and adolescents A systematic review $>$ Acesso em: 2 set. 2015 .

TACKETT, J. L., HERZHOFF, K., PAGE-GOULD, E., HARDEN, K. P., JOSEPHS, R. A. Personality X Hormone Interactions in Adolescent Externalizing Psychopathology. Personality Disorders: Theory, Research, and Treatment, v. 5, n. 3, p. 235-246, 2014. Disponível em: http://www.apa. org/pubs/journals/features/per-0000075.pdf. Acesso em: 10 set. 2015 .

WHO - World Health Organization. World Health Report 2002. Geneva, Switzerland; 2002.

WHO - World Health Organization. Global Status Report on Alcohol. Geneva, Switzerland; 2004.

WEITKAMP, K., DANIELS, J. K., ROMER, G., WIEGAND-GREFE, S.: Health-related quality of life of children and adolescents with mental disorders. Health and quality of life outcomes, v. 11, n. 129, 2013. Disponível em: http://www.ncbi.nlm.nih.gov/pmc/articles/PMC3733630. Acesso em: 3 set. 2015.

ZIMA, B. T., BUSSING, R., TANG, L., ZHANG, L., ETTNER, S., BELIN, T. R., WELLS, K. B. Quality of care for childhood attention-deficit/hyperactivity disorder in a managed care medicaid program. Journal of the American Academy of Child and Adolescent Psychiatry, v. 49, n. 12, p. 1225-1238, 2010. Disponível em: <http://www.ncbi. nlm.nih.gov/pubmed/21093772> Acesso em: 10 set. 2015. 